\title{
Evaluación de calidad en la educación básica: la entrada de la empresa en la escuela pública mexicana
}

\section{Gustavo Adolfo Enríquez Gutiérrez*}

Resumen. En este artículo se examina la evaluación de la calidad en la educación básica mexicana como operador social y político de corte liberal sobre la enseñanza de los docentes, el aprendizaje de los estudiantes y el vínculo con la gestión escolar en la educación básica. El texto permite discutir esta instrumentación a través de la problemática educativa generada por este enfoque y el desconocimiento del marco institucional de las escuelas públicas. El argumento cierra con la reflexión sobre la parcialidad del concepto de evaluación de calidad educativa y su incapacidad de vincularse con la problemática escolar. Recalca la evaluación como una herramienta de diálogo, análisis y cambio social con el fin de reposicionar a la escuela pública como espacio de construcción de políticas democráticas y comunidades autónomas.

Palabras clave: calidad educativa, políticas educativas, evaluación, propuestas educativas, México.

* Profesor investigador de la Universidad Pedagógica Nacional-Unidad 171, Morelos.

Una versión preliminar de este texto se publicó en Saber en la complejidad. 


\section{Quality Assessment in Basic Education: The entry of business into Mexican public schools}

Abstract. This article examines the quality assessment of Mexican basic education as a liberal social and political actor in terms of the training of teachers, the learning by students and the linkages between school management in basic education. The study allows for a discussion of the instrumentation through the educational problematic created by this focus and the lack of awareness of the institutional framework of public schools. The debate closes with a reflection on the bias inherent in the concept of educational quality evaluation and its inability to be linked with the schooling problematic. Evaluation is emphasized as a tool for dialog, analysis and social change with the aim of repositioning the public school as a space for the construction of democratic policies and autonomous communities.

Keywords: educational quality, educational policies, evaluation, educational proposals, Mexico. 


\section{Problema: evaluación de la calidad versus realidad escolar}

En términos generales, la calidad educativa expresa el interés del gobierno mexicano por intervenir con políticas educativas desde un enfoque unitario, fragmentario y descontextualizado de la situación que priva en las escuelas. De este modo, se desconocen las tareas escolares y el ejercicio específico de las instituciones educativas públicas en México (Aboites, 2013; Díaz, 2013; Coraggio, 1997; Franco, 1996; Olaf, 2013; Rockwell, 2013). Cabe reconocer, en este entramado de intereses, la existencia de múltiples tendencias, tensiones y niveles en la ejecución de estas políticas que matizan su concreción en las escuelas y dejan espacios e interdicciones donde es posible generar políticas contestatarias.

La calidad es la expresión de un adjetivo que concentra la urdimbre de disposiciones político-educativas entre la lógica de las actividades productivas, la problemática educativa, la situación escolar, las actividades docentes, los conflictos estudiantiles, el contexto de las escuelas y las comunidades (Aboites, 2012a; Díaz, 2005; Coll, 2013). Esta red de significado da diferentes sentidos a la operatividad de la calidad a partir del lugar que ocupan los actores sociales y su participación en el escenario educativo, lo que genera imágenes distintas sobre este aparente dominio y cerrazón ideológica. La calidad educativa se contrapone a la realidad escolar haciendo de la eficiencia, la eficacia, la pertinencia y la equidad una parte de la realidad dibujada desde la conformación de la empresa escolar sin contemplar las causas y problemas de las escuelas, los docentes, los niños, las madres y los padres de familia de las diferentes comunidades del país.

Las políticas educativas dominantes buscan reducir la intervención del Estado tanto en los subsidios como en el apoyo del gasto social a fin 


\section{Gustavo Adolfo EnRíQuez GutiÉrRez}

de hacer eficientes las instituciones educativas, la gestión escolar y el trabajo de los docentes. El eje operativo en la evaluación es el resultado de los exámenes como medio de acceso y permanencia de los estudiantes, medidos a través de calificaciones y colocando la calidad de los docentes como idónea o no idónea. El examen apoyado en leyes nacionales deja al margen la variedad de problemas sociales, pedagógicos y culturales del mundo escolar.

De acuerdo con José Luis Calva (23 de febrero de 2007), las políticas neoliberales buscaron la «aplicación del decálogo de «reformas estructurales〉 $\mathrm{y}$ 〈disciplinas macroeconómicas〉 recomendadas por los organismos financieros internacionales al mundo en desarrollo, que fueron sintetizadas en el «Consenso de Washington` por John Williamson», hace casi tres décadas. Estas políticas liberales parten del recorte al gasto social y a la mínima intervención del Estado para compensar esos gastos e incrementar el índice de recuperación económica a través de la participación social. Estos planteamientos se expresan en las políticas educativas de los últimos seis gobiernos señalando su interés por desarrollar las tesis neoliberales en las tareas educativas, en el papel del maestro y en el aprendizaje de los alumnos en el salón de clases, dejando de lado la complejidad del mundo escolar: la enseñanza de los docentes y el aprendizaje de niños, niñas y adolescentes. El discurso del gobierno pasó de indicar temas como la equidad y democracia social al de la eficiencia en las tareas de enseñanza, el aprendizaje en las escuelas y la gestión escolar, aspectos que aparecen como observables en la enseñanza eficaz y medibles en la evaluación del aprendizaje. Discurso centrado en la gestión del problema sin atender a las causas esenciales de la problemática. Este aparente embudo y dominio legal curiosamente replantea el papel de la escuela, el directivo, el docente, 
el alumno y la comunidad en nuevas narrativas desde las cuales es posible generar un espacio democrático y autónomo de participación social sobre el aprendizaje y la enseñanza. En otras palabras, reposicionar la política escolar desde las escuelas a partir de su situación, sus múltiples conflictos, y reconocer las diferentes propuestas colectivas elaboradas por el magisterio nacional a fin de poner al centro un proyecto escolar autónomo.

Una tarea necesaria es la reflexión colectiva sobre las tesis centrales de las políticas educativas actuales, el modelo económico y administrativo de la evaluación en la tarea docente y la forma en que la escuela se transformó en una empresa social. De ahí el interés por hablar de calidad, productividad y eficiencia en sus funciones (Aboites, 1997, 2012b; Foro Sevilla, 2012; Hernández, 2011). De ese modo, el objeto educativo se parcializa y fragmenta con el propósito de cumplir con una lógica de resultados y productos a través de pruebas de aprendizaje en los estudiantes, con exámenes a los maestros y desde la eficacia en la gestión de la escuela a través del cumplimiento de indicadores. Los instrumentos de evaluación —el examen, por ejemplo — aparecen como resultados del aprendizaje, la eficiencia en la consecución de objetivos y el uso racional de recursos (Gimeno Sacristán, 2002). El aprendizaje de los alumnos y sus intereses, necesidades y problemas, la ineficiencia en asignación de apoyos y la situación económica, cultural, política y social, se anulan con miras a identificar resultados arbitrarios ajenos al contexto del aula, la escuela y la comunidad.

El nuevo lenguaje de la política educativa desplaza términos como «formación» $\mathrm{y}$ «mejora de la educación» para hablar de la «calidad educativa» asociada a la empresa. Los resultados a través de evaluaciones, pruebas y exámenes manifiestan la calidad de la empresa educativa mediante la eficacia en la función educativa, en la tarea docente y el aprendizaje de 
los estudiantes. Este argumento aísla el contexto social concreto de las escuelas, generalizando sus errores y parcializando sus dificultades. Los parámetros generales de la calidad se aplican a todo el país mientras se desconocen las especificidades de los docentes y las escuelas. La escuela vista como empresa parte de un modelo de eficacia social y calidad que fragmenta la comprensión de las tareas de aprendizaje y enseñanza. De ahí la necesidad de colocar la evaluación educativa como un eje de reflexión colectiva en la construcción de políticas educativas democráticas desde la escuela, los docentes y los alumnos; poniendo de relieve el papel de la educación en la sociedad y la fragmentación argumental de la razón empresarial en el aprendizaje de niños, niñas y jóvenes mexicanos. Con base en esas ideas, el artículo se organiza en dos apartados: en el primero se aborda el problema de la calidad en la educación bajo la pregunta ¿solución o problema educativo?; y en el segundo la cuestión de la evaluación, ¿examen o reflexión colectiva del aprendizaje?

\section{La calidad en la educación: ¿`solución o problema social?}

En este apartado, se discuten los presupuestos sobre la calidad educativa con el fin de debatir la idea de mejora que supone. De esa manera, se disgregan tres aspectos generales que conforman el modelo de calidad educativa: las instituciones internacionales y nacionales que sugieren su adopción, los agentes académicos y consultores encargados de establecer sus indicadores y la forma en que se concreta el modelo con instrumentos de evaluación. Tales aspectos permiten entender el corte del entorno de las escuelas y el embeleco en las demostraciones acerca de su calidad. La calidad de la educación se 
impone gradualmente en la educación al comienzo del siglo pasado con la pedagogía industrial de Bobbit en Estados Unidos (Díaz, 1988; 2005) y se entreteje con las políticas de los organismos internacionales como la Organización de las Naciones Unidas para la Educación, la Ciencia y la Cultura (UNESCO, por sus siglas en inglés), la Organización para la Cooperación y el Desarrollo Económicos (OCDE), el Banco Mundial (BM) y la Organización de Estados Americanos (OEA). En fechas recientes se instrumentan estas políticas en instituciones nacionales como la Secretaría de Educación Pública (SEP) y el Instituto Nacional para la Evaluación de la Educación (INEE) (Martínez, 2004). En el caso mexicano, el cruce entre sugerencias internacionales, políticas de gobierno y la intervención de instancias académicas, políticas y empresariales concreta la calidad educativa en las instituciones escolares. Un ejemplo de instrumentación político-académica de estas ideas es el Centro de Estudios Educativos (CEE) que en los años noventa del siglo XX estableció criterios sobre la manera de operar la calidad en la educación. Aunado a lo anterior, estas políticas de la calidad toman cuerpo en las ideas de investigadores, académicos y especialistas en el terreno educativo. El trabajo de Silvia Schmelkes (1994) es un caso emblemático en el que el discurso académico se entreteje con el político. A continuación, se indican puntos generales a fin de comprender el tránsito de este concepto y su impacto en la conformación de la lógica empresarial en el terreno educativo de México.

La Declaración Mundial sobre la Educación para Todos y el Marco de Acción para Satisfacer las Necesidades Básicas de Aprendizaje, a través de la Conferencia Mundial de Educación para Todos en Jomtien, Tailandia (1990), planteó el aprendizaje como una exigencia social. En este sentido, el aprendizaje fue un eje del desarrollo social y una necesidad básica apoyada 


\section{Gustavo Adolfo EnRíQuez GutiÉrRez}

por los gobiernos a fin de que niños, niñas y jóvenes contaran con las herramientas y contenidos esenciales para sobrevivir, trabajar, participar, mejorar la calidad de su vida, tomar decisiones y continuar estudiando (UNESCO, 1990). El aprendizaje se convirtió en una herramienta básica de lectura, escritura, expresión oral, cálculo y resolución de problemas. En otras palabras, las necesidades básicas de aprendizaje facilitaron integrar a la población joven a la sociedad, le dotaron de herramientas esenciales en su participación social y trabajo. El aprendizaje desde esta perspectiva fue una obligación colectiva de los jóvenes en aras de contar con los artefactos de trabajo, integración y conocimiento permanente; sin embargo, las actividades comunitarias, el aprendizaje autónomo y el ocio productivo no fueron considerados importantes. La única opción posible fue la escolarización y la institución escolar occidental (Illich, 2015).

Por su parte, la OCDE (1991) dio a conocer la situación de la educación mundial con Escuelas y la calidad de la enseñanza. Informe Internacional. ${ }^{2}$ Este informe plasmó los problemas y las recomendaciones sobre la escuela y la enseñanza en el nivel internacional. La calidad sobre la educación apareció como marco de referencia operativo internacional sobre las políticas educativas. Entre los planteamientos señalados por la OCDE, el término calidad adoptó múltiples significados, pero tres direcciones lo enmarcan: subjetivo, descriptivo y normativo. A pesar de la variedad de significados, la OCDE señaló cuatro orientaciones: a) atributo específico, b) grado de excelencia o valor relativo, $c$ ) lo bueno o excelente y $d$ ) juicios no cuantificados

${ }^{2}$ De la apropiación del conocimiento escolar de los estudiantes, su aprendizaje, la calidad pasa a la identificación de los procesos desarrollados por los docentes, su enseñanza. La enseñanza, las estrategias, las actividades y las formas de organización de clase forman el eje de identificación de la calidad de las escuelas. El docente es el centro del trabajo escolar y de la recepción de información de los estudiantes como parte de la tarea de enseñar. 
(OCDE, 1990). La OCDE indicó la obligatoriedad de conocer los atributos y el valor de la educación, y de emitir juicios sobre su impacto social a través de la evaluación. La calidad educativa implicó conocer su marca social y valor sin contemplar la complejidad de los contextos socioculturales donde se concreta el aprendizaje (Belgich, 2007; Enkvist, 2003; Skliar, 2002; Tomlinson, 2005).

En el marco de la Conferencia de la UNESCO y el Informe de la OCDE, Schmelkes (1994), como parte de la OEA, recuperó estos planteamientos y señaló la calidad como la capacidad de proporcionar a los alumnos de educación básica los códigos culturales básicos que les ayuden a su participación democrática como ciudadanos, resolver problemas, seguir aprendiendo y desarrollar valores y actitudes sociales. Desde esta mirada, los códigos culturales básicos permitieron la participación democrática, la ciudadanía y la integración social. Un ciudadano, desde esta perspectiva, buscó una vida de calidad tanto al resolver problemas como al aprender constantemente. La calidad se identificó con las «necesidades» enseñadas en la institución escolar y usadas en la vida social a fin de resolver problemas, planear soluciones, mejorarlos y tener un liderazgo claro. Además, se reconocieron aspectos como la autocrítica, el valorar la diversidad, el consenso, la justicia y la responsabilidad. De esta manera, la calidad fue establecida como un comodín político que hizo las veces de base común de intervención y forma de emitir juicios sobre la educación nacional. Implícitamente, la evaluación de resultados fue, a su juicio, la forma de conocer la pertinencia de la operación de las políticas educativas y el índice palpable de su relevancia social.

En el caso de las políticas educativas mexicanas, la calidad en la educación tuvo como antecedente la modernización educativa presentada en el Programa para la Modernización Educativa (PME) que en 1989 mencionó: 


\section{Gustavo Adolfo EnRÍQuez GutiÉRREZ}

La característica distintiva de la educación moderna debe ser la calidad. Para lograrla, se propone revisar los contenidos, renovar los métodos, privilegiar la formación de maestros, articular los diversos niveles educativos y vincular los procesos pedagógicos con los avances de la ciencia y la tecnología (Poder Ejecutivo Federal, 1989:19).

El PME y el CEE (1994) establecieron el marco político y educativo de la calidad educativa a partir de la eficiencia en el logro de objetivos, la eficacia en el uso de recursos, la pertinencia de los aprendizajes y la equidad en la distribución de recursos. Estas ideas fueron señaladas por Schmelkes (1990) cuatro años antes como retos de la modernización en educación básica. Desde su visión, la calidad educativa supuso relevancia de los objetivos y contenidos, así como eficacia en su dominio a fin de abarcar un mayor número de alumnos. La calidad demandó acceso, permanencia y logro de objetivos, además de equidad y eficiencia: «hacer más con menos». La calidad se apoyó de una racionalidad técnico/administrativa apegada a la valoración económica de la educación que desarticuló la realidad escolar, la cultura de la escuela y las condiciones del aprendizaje cotidiano del salón de clases (Escolano, 2007; Glazman, 2005; Mercado, 2012; Rockwell, 2013).

Felipe Martínez Rizo (1996), académico universitario de Aguascalientes y uno de los principales promotores de la calidad educativa en la educación básica en México, esbozó este concepto como «sistémico», considerando el entorno a través de recursos, insumos, procesos, productos, objetivos y necesidades vinculadas con la eficiencia y la eficacia interna y externa. La eficiencia resultó al comparar productos del sistema con los insumos que se utilizaron. La eficacia interna se refirió a la coincidencia 
de los productos con los objetivos planeados y la externa se entendió al contrastar si los productos respondieron a las necesidades exteriores al sistema. Un último aspecto relacionado con lo anterior fue la relevancia o correspondencia de los objetivos con las necesidades del entorno. Tanto Schmelkes (1990) como Martínez (1996) colocaron la calidad educativa a partir de objetivos sociales relevantes y las condiciones sociales de igualdad para todos los estudiantes de la manera más económica posible, sin tocar las condiciones estructurales en que la institución escolar se desarrolla en cada contexto particular (Dubet, 2010; Rockwell y Quiroz, 1990). La empresa escolar racionaliza sus funciones sin asentar sus argumentos en las personas que le dan vida y en las potencialidades de todo proceso de aprendizaje y enseñanza: la autonomía de las instituciones y la creación de las personas.

En relación con los planteamientos anteriores, Carlos Muñoz Izquierdo (1999) consideró el concepto de calidad de la educación bajo dos adjetivos: normativo y multifactorial. Además, lo integró en cinco dimensiones: 1. Pedagógica: eficacia. Lograr las finalidades de los planes de estudio. 2. Filosófica: relevancia. Los objetivos y acciones educativas responden a intereses sociales. 3. Cultural: pertinencia. Educación adecuada tanto a los individuos como a la sociedad. 4. Social: equidad. Distribución equitativa de oportunidades en la sociedad. 5. Económica: eficiencia. Aprovechamiento de recursos. Desde su mirada, la educación de calidad fue resultado de la eficacia en la distribución equitativa en la sociedad, al alcanzar relevancia y pertinencia cultural aprovechando óptimamente los recursos en su impartición (Izquierdo, 1999).

Estas ideas sobre la calidad de la educación cobran materialidad en el sexenio de Vicente Fox (2000-2006) a través de la creación del Instituto 
Nacional para la Evaluación de la Educación (INEE) ${ }^{3}$ en 2002 y con la Alianza por la Calidad de la Educación (ACE) en 2007. Desde la perspectiva de la ACE, la problemática de la educación toca dos puntos generales señalados en la calidad de la educación: cobertura y eficiencia terminal. En la ACE se señaló: «[El] imperativo [de] hacer de la educación un factor de justicia y equidad, fundamentado en una vida de oportunidades, desarrollo integral y dignidad para todos los mexicanos» (SNTE/Gobierno Federal/SEP/ SHCP/Sedesol/Salud, 2007:5). Además de cobertura, la eficiencia se logró al tomar en cuenta cinco dimensiones: 1. Modernización de los centros escolares. 2. Profesionalización de los maestros y de las autoridades educativas. 3. Bienestar y desarrollo integral de los alumnos. 4. Formación integral de los alumnos para la vida y el trabajo. 5. Evaluar para mejorar. En este mismo sentido, el Programa Sectorial de Educación SEP 2007-2012 expresó:

Los criterios de mejora de la calidad deben aplicarse a la capacitación de los profesores, la actualización de programas de estudio y sus contenidos, los enfoques pedagógicos, los métodos de enseñanza y recursos didácticos (...) La evaluación será un instrumento fundamental en el análisis de la calidad, la relevancia y pertinencia del diseño y operación de las políticas públicas en materia de educación (DFCMS, 2008:15).

De esta manera, se utilizó parcialmente la evaluación como un instrumento de valoración de las políticas públicas a través de exámenes aplicados

\footnotetext{
${ }^{3} \mathrm{El}$ artículo $4^{\circ}$ del decreto de creación de INEE 2002 plantea sus funciones e indica como primera orientación: «Buscará alcanzar la mayor calidad en el desarrollo de modelos e instrumentos de evaluación, atendiendo la confiabilidad y validez, en todas sus dimensiones».
} 
a estudiantes en México. ${ }^{4}$ Ejemplos de estos exámenes fueron los Exámenes de Calidad y Logro Académico (Excale) en 2003 y la Evaluación Nacional del Logro Académico en Centros Escolares (ENLACE) en 2005 a través del Programa de Escuelas de Calidad (PEC) de 2001, además de la evaluación de la enseñanza a los maestros con el Programa Nacional de Carrera Magisterial (PNCM) en 1993. Esta visión estrechó las miras sobre el aprendizaje y la enseñanza a partir de los resultados en pruebas sin colocar las trayectorias, la historia, las experiencias y el contexto socioeducativo, económico-cultural de estudiantes y maestros (Aboites, 2012b; Cole, 1999; Berteley, 2013; Gardner, 2011; Jiménez, 2012). La mirada constreñida de un instrumento evaluativo no puede dar cuenta de la totalidad del mundo escolar vivido, sentido, pensado y actuado por estudiantes, docentes, directivos y padres de familia.

La OCDE (2012) estableció a la educación como eje del desarrollo económico y social. La educación se propuso como una palanca de cambio social y personal. De tal suerte que la calidad fue materializada a través de la enseñanza eficaz, el resultado de exámenes, la gestión y el liderazgo escolar. La calidad se instrumentó en la enseñanza a través de estándares sobre la práctica docente, programas de formación pertinentes, «autonomía» escolar y financiamiento. La calidad del aprendizaje de los estudiantes se identificó con exámenes estandarizados, la enseñanza eficiente se midió con estándares docentes y el liderazgo escolar a través de estándares

\footnotetext{
${ }^{4}$ En principio los exámenes se centraron en el aprendizaje de los alumnos: las formas en que éstos conocían y respondían a la información que los docentes transmitían en clase, centrando su tarea en la cantidad de respuestas correctas dadas en las evaluaciones realizadas con este instrumento. Después, este instrumento se cambió a la enseñanza del profesor: la manera como el docente contesta preguntas sobre su forma de propiciar la mediación de información dada a los alumnos en clase.
} 


\section{Gustavo Adolfo EnRíQuez GutiÉrRez}

de gestión. La calidad sirvió de marco en la operación de las políticas del gobierno mediante exámenes fuera del nicho social, cultural y educativo en que se realizaron la enseñanza y el aprendizaje escolar de las escuelas del país.

La calidad en la educación establece tensiones en el discurso educativo desde la tarea docente como la variable causal del aprendizaje sin contemplar su complejidad, especificidad, historia y contexto. La calidad no atiende a los problemas de enseñanza y aprendizaje que se presentan en las diferentes situaciones escolares, por lo que pondera el trabajo individual, como señala un maestro: «La Ley General del Servicio Profesional Docente, sí tiende más a individualizar, o sea, preocúpate maestro por hacer bien tu trabajo, porque la evaluación es individual» (Juncos, 2016:397). Aprender y enseñar son procesos relacionados, pero su conexión no es automática. El disfrute, la incertidumbre o la angustia forman parte de este proceso. La escuela es un ámbito de los múltiples de aprendizaje en que los niños conocen, descubren e indagan; en este sentido, un maestro explica: «Si los chicos que van a la escuela no son felices, nos está llevando la fregada» (Juncos, 2016:397). La escuela no es el centro de la vida, debe reorganizarse a fin de permitir vivir, disfrutar y crear un espacio de juego, diversión y aprendizaje. La vida de los estudiantes mexicanos promedio está plagada de dificultades individuales, sociales, económicas, culturales y lingüísticas que afectan su aprendizaje, y que las políticas del Estado mexicano poco modifican (Aboites, 2012a, 2012b y 2013; Berteley, 2013 y 1992; Cole, 2003; Illich, 2005; Foro Sevilla, 2012; Rockwell, 2013).

La institución escolar no tiene y no puede tener el monopolio del conocimiento, basta indicar que: «Sólo 72 por ciento de los estudiantes cursa la educación primaria en seis años; esta proporción es considerablemente 
menor (50 por ciento) entre quienes asisten a escuelas indígenas» (INEE, 2012:3). Así, 30 por ciento de la población global y 50 por ciento de la población indígena no asisten a las escuelas, aprenden fuera de los ámbitos de la institución escolar. En consecuencia, estrechar la evaluación en exámenes a través de cajones fijos, deja de lado su parte creativa, lúdica e inquisitiva, lo que reduce el potencial de los estudiantes. La evaluación restringida a un instrumento la convierte en asunto técnico descontextualizado y personal; en este sentido, explica un maestro: «QQue van hacer el examen ahorita y el que no sirve se va! ¿Qué va a suceder? Va pasar lo mismo que en Carrera Magisterial: el maestro se va a poner a estudiar y va a trabajar para él y va a dejar a los niños» (Juncos, 2016:434).

La calidad medida a través de un examen se convierte en fiscalización del trabajo docente dejando de lado las diferencias, los problemas de fondo en la enseñanza y el aprendizaje. La enseñanza tomada aisladamente se desarticula de sus múltiples relaciones y de la realidad cotidiana en que se aprende y enseña en la escuela. El aprendizaje visto como almacén de información elimina la parte fundamental de la persona: el deseo de conocer, descubrir y comprender por sí mismo. La calidad fue colocada en el terreno educativo como parte de la pedagogía industrial de influencia pragmática a través del diseño de un conjunto de políticas educativas desarrolladas por instituciones internacionales y nacionales operadas por consultores nacionales. La calidad fue desarrollada como un concepto comodín definido de forma general hasta llegar a concretarse en la evaluación a través de exámenes desde los cuales se colocan respuestas como indicadores de información obtenida por los estudiantes y las respuestas correctas sobre la enseñanza en los docentes, sin atender a los problemas del aprendizaje, de la enseñanza y de las escuelas en estos casi 40 años de su predominio. 


\section{La evaluación: ¿el examen estandarizado o la reflexión colectiva del aprendizaje?}

En este apartado se discuten los supuestos sobre la evaluación de la calidad educativa y se cuestiona la idea de mejora que supone. Para ello se toman dos aspectos del modelo de evaluación: la enseñanza eficaz y el examen como indicador de la calidad. Estos aspectos permiten comprender la fragmentación de la evaluación y su falacia argumental en la exploración de las problemáticas de las escuelas públicas mexicanas y la falta de un trabajo colectivo en relación con el aprendizaje.

La evaluación desde el discurso político educativo fue modificando su interés analítico desde las tareas escolares, el aprendizaje de los alumnos, la organización del tiempo, espacio y materiales, pasando al terreno del trabajo académico y las funciones de enseñanza, los programas y planes de estudio, después a las instituciones escolares y, finalmente, a los sistemas educativos de un país. En este tránsito, un instrumento, el examen, se colocó como el portador de información objetiva, universal, expedida y medible. Formalizado por Binet y Simon en Francia a principios del siglo XX, con el fin de contar con información sobre la forma de solucionar problemas prácticos, se convirtió en parte fundamental de las decisiones educativas, sociales y políticas sobre la identificación, la clasificación y la jerarquización de los estudiantes en la escuela y un medio de tipificación sobre la normalidad, anormalidad o competencia de una persona (Binet y Simon, 1916; Padilla, 2012; Stenberg, 2003). Pasó de ser una parte del proceso de aprendizaje a convertirse en el medio principal de toma de decisión sobre la enseñanza y el aprendizaje, además de valorar al personal de una empresa, el trabajo de un profesionista, la promoción y la eficacia de una actividad. En el caso 
de la institución escolar, esta eficacia se concretó en la enseñanza, la enseñanza eficaz.

La enseñanza eficaz supone la actividad docente como un objeto delimitado, observable y medible a través de estándares sobre los valores, las habilidades y los conocimientos de una buena enseñanza. Brophy (2000) estableció 12 principios para la enseñanza, en un material publicado por la SEP: a) ambiente de apoyo en el salón de clase, b) actividades relacionadas con el currículo y la organización de clase, c) alcanzar los objetivos y propósitos educativos, $d$ ) aclarar aprendizajes esperados y estrategias de aprendizaje, $e$ ) contenidos coherentes, $f$ ) diálogo reflexivo, $g$ ) actividades prácticas, $h$ ) compromiso de los estudiantes en sus actividades, $i$ ) enseñar estrategias, $j$ ) aprendizaje colaborativo, $k$ ) evaluación enfocada a objetivos, l) logros del aprendizaje.

Esta serie de principios estableció un marco de referencia sobre el trabajo docente delineado como parte de la jerga académico-educativa sobre la enseñanza eficaz (Murillo, 2008; Román, 2011) y de las escuelas eficientes (INEE, 2015; UNESCO, 2004; OCDE, 2012). Se trata de elementos centrales del discurso político didáctico sobre las funciones de la enseñanza y de una escuela de calidad. Aspectos fundamentales, pero fuera del trabajo docente en la escuela, sus condiciones en las aulas y las diferentes situaciones del aprendizaje escolar.

En la tradición pedagógica, la enseñanza se definió desde la didáctica y su centro de articulación fue la pregunta ¿cómo enseñar? En la didáctica se relacionaron tres aspectos. Las tareas propias de los docentes: la enseñanza, las actividades de aprendizaje con los estudiantes y el contenido a trabajar en clase. Además de estos elementos, se integró un conjunto de aspectos que dieron sentido al sistema didáctico: funciones, tareas, actividades y efectos 


\section{Gustavo Adolfo EnRíQuez GutiÉrRez}

(Saint-Onge, 2000; Díaz, 1984). Este conjunto de reflexiones aportadas por la didáctica fue simplificado en las recomendaciones de organismos internacionales y en el informe Mejorar las escuelas. Estrategias para la acción en México (OCDE, 2012:75), donde se sugieren cinco estándares para la eficiente enseñanza: 1. Planeación: contenidos, objetivos, estrategias y evaluación elegidos y seleccionados. 2. Gestión del ambiente de clase: manejo del grupo y relaciones en el grupo. 3. Gestión curricular: conocimiento de la asignatura, sus relaciones y conexiones. 4. Didáctica: presentación del currículo, atención a la diversidad, organización del grupo, aprendizaje, relaciones, recursos, tiempo, indicaciones, preguntas y actividades. 5. Evaluación: valoración personal, entre alumnos, con el docente y del conocimiento.

Estos estándares formaron parte de la enseñanza eficaz en México e incidieron en la forma de evaluar el trabajo docente y en la evaluación de su desempeño, cuyos propósitos fueron:

a) Valorar el desempeño del personal docente de Educación Básica, para garantizar un nivel de suficiencia en quienes ejerzan labores de enseñanza, y asegurar de esta manera el derecho a una educación de calidad para niñas, niños y adolescentes.

b) Identificar necesidades de formación de los docentes de Educación Básica que permitan generar acciones sistemáticas de formación continua, tutoría y asistencia técnica dirigidas a mejorar sus prácticas de enseñanza y su desarrollo profesional.

c) Regular la función docente en la medida en que la evaluación del desempeño servirá de base para definir los procesos de promoción en la función y de reconocimiento profesional del personal docente de Educación Básica (SEP, 2015a:8) 
De este modo, las políticas educativas señaladas por el gobierno buscaron valorar, identificar y regular la enseñanza, y en este sentido, promover y reconocer la enseñanza de calidad además de generar una estrategia de formación de las necesidades de los docentes basada en tutorías. Vinculado con estas estrategias, se indicó el tipo de docente que se requería a través del perfil de egreso:

Un docente que conoce a sus alumnos, sabe cómo aprenden y lo que deben aprender; organiza y evalúa el trabajo educativo y realiza una intervención didáctica pertinente; reconoce como profesional que mejora continuamente para apoyar a los alumnos en su aprendizaje; asume las responsabilidades legales y éticas inherentes a su profesión para el bienestar de los alumnos y fomenta el vínculo de la escuela y la comunidad para asegurar que todos los alumnos concluyan con éxito su escolaridad (SEP, 2015b:14).

Las decisiones tomadas desde esta lógica no inciden en la solución de los problemas de enseñanza y su análisis sobre las dificultades de aprendizaje en las escuelas se fragmenta sin contemplar la dinámica, las relaciones y la entropía propia de una realidad compleja concretada en los salones de clase.

Tal es el caso del fracaso escolar de los niños en México, que tiene hondas raíces sociales, económicas y educativas. Cada niño y niña forma parte de la desigualdad educativa, tema necesario al momento de investigar el aprendizaje escolar de la infancia en México, por ejemplo: «La acentuada desigualdad en la distribución de la riqueza: mientras 21.2 millones de mexicanos viven en situación de pobreza alimentaria, 11.3 millones (10 por ciento más rico) concentran 38 por ciento del ingreso corriente nacional» 


\section{Gustavo Adolfo EnRíQuez GutiÉrRez}

(INEE, 2012:2). El papel de la educación y las necesidades sociales no aparecen claramente mencionados al abordar los cuestionamientos que se proponen, dejando de lado las desigualdades económicas, sociales, políticas, culturales y educativas de niños, adolescentes y jóvenes en México.

Como indicador de la calidad, el examen tiene una historia que partió de una porción de la psicometría estadounidense del siglo XX. Esta psicometría focalizó a este instrumento como fundamental en la detección de la normalidad, la discapacidad y la evaluación de la inteligencia a fin de clasificar a niños, jóvenes y adultos de acuerdo con la medición de su inteligencia. Absurdos científicos sobre la «pobreza intelectual» de las personas indicados en Estados Unidos por Hernstein y Murray (1994) a través de pruebas de inteligencia y la medición de cociente intelectual. Ambos señalaron a los angloamericanos como la porción racial con mayor inteligencia, a diferencia de los hispanos y africano-descendientes, tesis severamente cuestionadas por la psicología social y cultural actual que reflexiona sobre la inteligencia humana dependiente de la cultura, la sociedad, la geografía y la historia (Cole, 2003; Gardner, 1983).

En la actualidad, el examen o test es usado en instituciones educativas, empresas, organismos privados y públicos en la selección de personal, estudiantes o empleados. En el caso de la educación pública mexicana, la evaluación al docente se inició con el Examen de Ingreso a la Educación Normal (EIEN) en 1984 a fin de evaluar el ingreso de los aspirantes a las licenciaturas de las escuelas normales. En 1989 se aplicó el Instrumento de Diagnóstico y Clasificación para el Ingreso a la Educación Normal (IDCIEN); en 1993 con la creación del Programa Nacional de Carrera Magisterial (PNCM) se aplicaron los Exámenes Nacionales para la Actualización de los Maestros en Servicio (ENAMS); en 2008, el Examen Nacional de Conocimientos 
y Habilidades Docentes (ENCHD); y recientemente la Evaluación del Desempeño Docente (EDD) 2016 (SEP/OCDE, 2012). Estos instrumentos no han modificado la calidad de la enseñanza de los maestros, no dan cuenta de las causas de los problemas de enseñanza y no han modificado los bajos resultados, por ejemplo, en las escuelas comunitarias e indígenas del país (OCE, 2010; Tapia y Valentini, 2015; Tenti y Torres, 2000).

En estos 33 años, los exámenes aplicados a los docentes tuvieron tres sentidos: a) ingreso a la formación inicial, b) selección al servicio y c) evaluación de su actualización y desempeño. En todos estos casos, la enseñanza se concentró en conocimientos, habilidades y desempeños identificados a través de preguntas desde el espacio imaginado por los políticos, empresarios y funcionarios sobre la tarea docente. La formación permanente y sus implicaciones cotidianas en el trabajo educativo no aparecen, así como la cultura, la historia y factores sociales asociados a su trabajo concreto se desconocen. Al respecto, Tapia y Valentini manifestaron: «Los datos muestran que en el país operan fuertes mecanismos de reproducción social y cultural, y que la escuela sigue siendo una importante fuente de exclusión social, aun cuando se haya avanzado en la cobertura y la escolarización de la población» (2016:49-50). La escuela no responde a las necesidades sociales y tampoco a los intereses de los alumnos, Torres y Tenti señalaron: «¿Cuál es la parte más aburrida de la clase? Le preguntamos a una joven alumna después de salir de una Telesecundaria. «La parte del profesor`, respondió, seguida a coro por sus compañeros» (2000:50). En este mismo sentido, el video viral de Mars Aguirre en México expresó: «Estoy hasta la madre del pinche sistema retrógrado en el que hemos estado sometidos toda la vida» (2017). Un elemento adicional lo plantea George, el joven de preparatoria de la película The art of gettin by (El arte de pasar de todo), quien señala: 


\section{Gustavo Adolfo EnRÍQUez GutiÉRREZ}

Vivimos solos y morimos solos. ¿Por qué tengo que pasarme la vida trabajando, sudando y luchando?, ¿por una ilusión?, porque ni tener amigos o novia o realizar tareas como conjugar el pluscuamperfecto o calcular la raíz cuadrada de la hipotenusa me ayudarán a evitar mi destino (...) Tengo cosas mejores en las que emplear mi tiempo (Winesen, 2011).

Estas expresiones muestran el problema de la escuela y las formas en que ésta invierte sus esfuerzos por inculcar información y trabajo sin pensar en diferentes formas de generar el aprendizaje en los estudiantes (Enkvist, 2003; Illich, 205).

Estudios etnográficos, antropológicos y sociales han demostrado que el reconocimiento de las características socioculturales del aprendizaje de los niños es un factor necesario al momento de interactuar con los niños e impulsar su desarrollo (Bertely, 1992, 2012; Fuentes, 2013; Rockwell, 2013). En ese tenor, señaló el propio INEE:

Se estima que de cada mil niños que se inscribieron en primaria en el ciclo escolar 2001/2002, sólo 438 terminaron la educación media superior 12 años después. Las niñas siguen trayectorias más regulares: 465 de cada mil logran concluir la educación obligatoria a tiempo, mientras que 411 niños lo hacen (2012:3).

De ahí la necesidad de realizar estrategias de adaptación docente a los estilos y formas de aprendizaje de los niños, las niñas y las circunstancias sociales, culturales, intereses y realidad de las comunidades.

Los problemas de la enseñanza y el aprendizaje se centran en la manera de dosificar, transmitir y certificar conocimientos. Los problemas sociales del aula se descolocan de lugar y la aplicación de exámenes deja de 
comprender las razones que hacen que los docentes de comunidades indígenas, marginadas, rurales y migrantes aprendan, máxime que: «Un 44 por ciento de las escuelas del país es multigrado. Esta condición se presenta en todas las primarias comunitarias, en 30 por ciento de las generales y en dos de cada tres planteles indígenas. El 90 por ciento de las escuelas indígenas se ubica en localidades menores a 2 mil 500 habitantes» (INEE, 2012:6). La realidad del país se refleja en las escuelas donde prevalece la diversidad, en localidades dispersas y con una falta de reconocimiento a la cultura, la lengua y la autonomía de las localidades.

El examen es un elemento importante, pero no puede ser la única pieza a considerar en la enseñanza. En este sentido, Backhoff argumenta:

En un estudio realizado con información de PISA 2012, cuyo foco de atención fueron las matemáticas, los estudiantes mexicanos que tuvieron mayores oportunidades para aprender: Razonamiento matemático puro y Razonamiento aplicado, obtuvieron menores puntuaciones en PISA que aquellos que no tuvieron dichas experiencias. Asimismo, la información de PISA 2015, cuyo foco de atención fueron las Ciencias, señala que los estudiantes mexicanos mostraron mayor interés, gusto y motivación por estudiar temas relacionados con las Ciencias, respecto a los alumnos de los países de la OCDE (2017:2).

El examen da a conocer parte del proceso e indica aspectos que es necesario comprender en la mejora de la enseñanza, pero no puede ser un medio definitivo sobre el valor de la enseñanza y el aprendizaje de las instituciones educativas.

La forma de abordar el aprendizaje, la reprobación y la enseñanza, aparecen como asuntos individuales en una relación tangencial con lo social. Los 


\section{Gustavo Adolfo EnRÍQuez GutiÉRREZ}

exámenes pueden ser un poderoso aliado en el diseño de estrategias didácticas en el aula; sin embargo, requieren ser concebidos desde una perspectiva amplia de evaluación. La evaluación en una visión razonable-democrática ayuda a comprender lo que sucede en el aula, en otras palabras: ¿qué pasa en este espacio?, ¿cómo se enseña?, ¿cómo aprenden los estudiantes? y ¿qué formas de desarrollo personal se han generado? (Díaz, 1984; Casanova, 1998). Vista como medio de permanencia, promoción y reconocimiento, poco puede hacer en la comprensión de los problemas de aprendizaje y enseñanza.

Los docentes que trabajan con grupos vulnerables, escolares con bajo desempeño, estudiantes con discapacidad, son juzgados a través del resultado de un examen, al igual que el análisis de los puntajes de los exámenes de toda la población, sin comprender las dificultades de fondo. En este tenor, señala Andrés Sandoval:

Para dar un ejemplo, sabemos que el desempeño de México en ciencias está en 115 puntos por debajo del de Finlandia y 40 puntos por arriba del de Argelia; pero también sabemos que el ingreso per cápita de Finlandia es casi cinco veces más alto que el de México y que el de Argelia es 2 veces más bajo que el de México y 10 veces más bajo que el de Finlandia (2016:1).

El examen indica puntajes y resultados, pero deja de lado las causas, los problemas y los vacíos no resueltos por los examinados, como en su momento lo hizo Piaget, al examinar los resultados de los exámenes elaborados por Binet, éste formuló preguntas sobre sus causas y buscó nuevas formas de comprender la inteligencia de los niños (Ferreiro, 1999). La tarea inquisitiva de hurgar en la problemática se deja de lado a fin de identificar, clasificar y emitir juicios parciales sobre el valor de un estudiante o un docente. 
Las pruebas dicen partir del aprendizaje de los niños, pero éstas deben complementarse con otra información y en condiciones de mayor equidad social. Las escuelas públicas, al igual que las privadas, como explica Emmanuel Gama, no generan un ambiente de indagación sobre el aprendizaje escolar y la forma en que los ambientes influyen en éste: «Es muy difícil que un país progrese si la mayoría de su población no tiene competencias básicas, pero es imposible si sus élites no las tienen y sólo basan su privilegio en características que no generan un beneficio en su entorno» (Gama, 2016:3). El plantear el examen como instrumento nodal del aprendizaje crea una situación inequitativa al centrar sus valoraciones en un momento determinado y no en la totalidad del proceso (Tapia y Valentini, 2016; Slee y Weiner, 2001).

Los teóricos de la sociología educativa de los años setenta y ochenta del siglo XX escribían sobre cómo los factores económicos imponían sus formas de razonamiento en la escuela. Martín Carnoy decía:

Los fines del desarrollo pueden ser igualar el consumo y la condición social que provienen del trabajo, dar un grado superior de movilidad intergeneracional, tanto hacia arriba como hacia abajo, y otorgar a la gente mayor control en el proceso de toma de decisiones. Si la escolaridad formal —el modo como está estructurada en las sociedades capitalistas — no contribuye significativamente a estas metas, es desarrollo sólo por contribuir a la mayor producción y el mayor consumo totales (1997:18-19).

La escuela no está contribuyendo a la toma de decisión en una sociedad cada vez más polarizada y elitista donde los políticos, los empresarios, los dueños de los medios de comunicación de masas, los productores de 


\section{Gustavo Adolfo EnRíQuez GutiÉrRez}

tecnologías, los creadores de diversiones para las masas y los encargados de las grandes compañías controlan la política, la economía, la cultura y las instituciones sociales. Hoy, es necesario repensar la escuela y la forma en que sus tareas conforman la ciudadanía, la democracia, la autonomía y la creación fuera de estos márgenes impuestos.

A casi 40 años de distancia, la lógica del mercado y la certificación de conocimientos como estímulo para el avance personal prevalecen en la mirada de las políticas educativas y sociales instrumentadas por el gobierno mexicano. La evaluación de la calidad educativa centrada en un informe, un expediente, un examen y en la planeación simplifica el compromiso, la intuición, la experiencia, la sensibilidad y el arte de enseñar tan necesarios en contextos complejos como los que se viven actualmente en las escuelas públicas mexicanas.

Una tarea pendiente en la construcción de una escuela democrática es la conformación de un proyecto de autonomía, democrático y crítico donde las personas — docentes, estudiantes y directivos - definan un nuevo rumbo a la educación. Una escuela donde estén puertas y ventanas abiertas a la vida, a una pedagogía de la experiencia, del aprendizaje autónomo y de un potencial a la invención de nuevos rumbos al aprendizaje. Crear un proyecto auténticamente colectivo desde y para la escuela pública. Tarea asentada en el trabajo común de todos, en nuevas formas de convivencia, en distintas maneras de elaborar el texto escolar de las escuelas de acuerdo con cada contexto escolar. El trabajo es de todos, en conjunto, sin tener una meta acabada, pero sí el compromiso de rehacer constantemente la participación de todos. Trabajo colectivo, donde cada quien tenga un lugar, un lugar para todos, donde todos dialoguen, escuchen y propongan en la nosotredad del respeto a cada uno y a todos. Tarea política de todos 
y por todos, en un hacer, rehacer interminable. En una discusión sobre el aprendizaje de niños, niñas y jóvenes desde una forma de evaluar que invite al diálogo, la comprensión, el análisis, la discusión, la formulación de propuestas, la mejora desde cada uno de los participantes. La evaluación es una tarea compleja reducida a un instrumento desde la lógica de la ganancia sin comprender la importancia de su reflexión conjunta como ejercicio abierto al conocimiento y al saber del grupo escolar.

\section{Reflexiones finales: el proyecto de autonomía escolar}

La calidad educativa se importa desde la empresa al terreno educativo. Este concepto parcializa el trabajo escolar ubicando la enseñanza como pieza clave del aprendizaje sin articularla con el sistema educativo, la institución escolar, la comunidad, la familia, los estudiantes y las relaciones con la cultura, la política, la economía y la propia naturaleza de la educación. La calidad es un adjetivo escurridizo, difícil de materializar, sobre todo al tomar cuerpo en la enseñanza de un maestro particular y de una escuela. La calidad impuesta desde un lugar pondera esta explicación e impide comprender otras formas de hacer, pensar y sentir.

El buen maestro aparece como imagen de un espejo doble y como tal es una imagen. No es la realidad desarrollada en las escuelas, donde los conflictos, la violencia, la falta de incentivos, las dificultades sociales rodean a la enseñanza e imponen matices al aprendizaje de los alumnos. Aplicar en cuatro horas un examen y responsabilizar del resultado al docente es más redituable que preguntar sobre los problemas sociales que afectan el aprendizaje e inciden en la enseñanza. Es decir, sobre las dificultades que 


\section{Gustavo Adolfo EnRíQuez GutiÉrRez}

implica trabajar en contextos marginados y de alta violencia, lugares en donde la corrupción, la desnutrición, los salarios exiguos y la falta de servicios influyen en los intereses de niños, niñas y se imponen contundentemente. En este mundo es necesario investigar, indagar y comprender a fin de diseñar estrategias de enseñanza y aprendizaje contextualizadas.

La evaluación como capacidad de diálogo desaparece al privilegiar resultados individuales y estadísticas sin dar cuenta de las causas de los problemas de enseñanza y aprendizaje que padecen las escuelas mexicanas. El análisis de las relaciones entre fracaso, pobreza, enseñanza y aprendizaje no se profundiza ni tampoco sus múltiples consecuencias. La evaluación requiere ser colocada como una reflexión social sobre las dificultades existentes en el aula con los niños y las niñas, y en el caso de las escuelas resulta aún peor identificar sus vínculos sociales, culturales, económicos y políticos con aquellos. La evaluación desde el diálogo, la participación, la comprensión, la estrategia de cambio y la construcción colectiva busca dar soluciones dinámicas, contextuales y parciales a los problemas. Es necesario situar a la escuela como generadora de políticas democráticas desde la base a fin desarrollar un espacio democrático que ayude al desarrollo de aprendizajes autónomos a partir de la experiencia de los maestros, desde el conocimiento de niñas, niños y padres de familia. La evaluación de la calidad es un armazón que necesita ser reflexionado colectivamente desde una teoría social de la evaluación que contemple su complejidad, diversidad y particularidades de la mano de sus actores principales: estudiantes, docentes y comunidades. Las políticas son un asunto público y las políticas del aprendizaje y la enseñanza son asunto de los actores educativos desde el salón de clases, la escuela, la comunidad y la sociedad, en otras palabras, políticas públicas desde la escuela fuera del razonamiento costo-beneficio de la empresa. 


\section{Referencias}

Aboites, Hugo (1997), Viento del norte: TLC y privatización de la educación superior, México, Universidad Autónoma Metropolitana/Plaza y Valdés. (2012a), «Los cimientos de la nueva evaluación», en La medida de una nación: los primeros años de la evaluación en México: historia de poder, resistencia y alternativas (1980-2012), México, Universidad Autónoma Metropolitana, pp. 39-293. (2012b), «El derecho a la educación en México. Del liberalismo decimonónico al liberalismo del siglo XX», Revista Mexicana de Investigación Educativa, 17(57), pp. 361-389.

(2013), «Implicaciones de la reforma educativa de 2012 al artículo 3ero constitucional», El Cotidiano (179), pp. 27-42.

Aguirre, Mars (2017), La Mars, chica deja la prepa, México, en https://www. youtube.com/watch?v=o2PzgRvlgg

Backhoff, Eduardo (2017), «Lo que los resultados de PISA no pueden explicar. Opinión 9 de enero», Educación Futura, pp. 1-2.

Belgich, Horacio (2007), Reflexiones sobre la práctica docente en los procesos de integración escolar, Buenos Aires, HomoSapiens.

Bertely Busques, María (2013), «Capítulo 1. Debates conceptuales sobre la educación multicultural e intercultural», en Multiculturalismo y educación 2002-2012, México, Consejo Mexicano de Investigación Educativa, pp. 41-79.

(1992), «Adaptaciones docentes a una comunidad mazahua», Nueva Antropología. Revista de Ciencias Sociales (42), pp. 101-120.

Brophy, Jere (2000), Enseñanza, México, Centro Nacional de Evaluación para la Educación Superior/Centro de Investigación y de Estudios Avanzados/ 


\section{Gustavo Adolfo EnRÍQuez GutiÉRREZ}

Consejo Mexicano de Investigación Educativa/Instituto Nacional para la Evaluación de la Educación/Universidad Politécnica Nacional/Impuesto sobre Actividades Económicas/ Institute for Better Education.

Binet, Alfred y Theodore Simon (1983), The development of intelligence in children, New Hampshire, Ayer Company, en http://www.indiana.edu/ intell/ binet.shtml.Binet

Calva, José Luis (23 de febrero de 2007), «El modelo neoliberal», El Universal.

Carnoy, Martin (1977), La educación como imperialismo cultural, México, Siglo XXI.

Casanova, María Antonia (1998), La evaluación educativa. Escuela básica, México, Secretaría de Educación Pública.

Centro de Estudios Educativos (CEE) (1994), «Editorial. Las investigaciones del CEE sobre la calidad de la educación básica», Revista Latinoamericana de Investigación Educativa, XXIV(1 y 2), pp. 5-11.

Cole, Michael (2003), Psicología cultural, España, Morata.

Coll Lebedeff, Tatiana (2013), «La reforma educativa, el poder del Estado y la evaluación», El Cotidiano (179), pp. 43-54.

Coraggio, José Luis (1997), «Las propuestas del Banco Mundial para la educación: ¿Sentido oculto o problema de concepción?», en José Luis Corraggio y Rosa María Torres (coords.), La educación según el Banco Mundial, Buenos Aires, Miño y Davila, pp. 11-74.

Díaz Barriga, Ángel (1984), Didáctica y currículo. Convergencias en los programas de estudios, México, Nuevomar. (1988), «Calidad de la educación. ¿Un adjetivo más de la política educativa?», Cero en Conducta (3), pp. 11-12.

(2005), El docente y los programas escolares. Lo institucional y lo didáctico, México, Pomares. 
(2013), El examen: textos para su historia y debate, México, Plaza y Valdés.

Dirección de Formación Continua de Maestros en Servicio (DFCMS) (2008), Prioridades y retos de la educación básica. Curso Básico de Formación Continua. Alianza por la Calidad de la Educación. Ciclo escolar 2008-2009, México, Sindicato Nacional de Trabajadores de la Educación/Gobierno Federal/Secretaría de Educación Pública/Secretaría de Hacienda y Crédito Público/ Secretaría de Desarrollo Social/Salud.

Dubet, Francois (2010), «Crisis de la transmisión y declive de la institución», Política y Sociedad, 47(2), pp. 15-25.

Enkvist, Inger (2003), La educación en peligro, Argentina, Ovejero Martín.

Escolano, A. (2007), La cultura material de la escuela, España, Berlanga/Centro Nacional de Información y Comunicación Educativa.

Ferreiro, Emilia (1999), La vigencia de Jean Piaget, México, Siglo XXI.

Franco, R. (1996), Los paradigmas de la política social en América Latina, Comisión Económica para América Latina y el Caribe.

Foro Sevilla (2012), Manifiesto por otra política educativa. La escuela pública de todos para todos, España, Morata.

Fuentes Molinar, Olac (2013), «La tarea del maestro y los desafíos de la evaluación docente», en La reforma constitucional en materia educativa: alcances y desafíos, México, Senado de la República/Instituto Belisario Domínguez, pp. 17-34.

Gama, Emmanuel (31 de agosto de 2016), «Los incentivos de las escuelas particulares para no generar educación de calidad», Nexos, pp. 1-3.

Gardner, Howard (1983), La teoría de las inteligencias múltiples, España, Paidós. Gimeno Sacristán, José (2002), La pedagogía por objetivos: obsesión por la eficacia, España, Morata. 


\section{Gustavo Adolfo EnRÍQuez GutiÉRREZ}

Glazman Nowlaski, Raquel (2005), «Orientaciones pedagógicas y sociopolíticas de la evaluación», en Las caras de la evaluación educativa, México, Universidad Nacional Autónoma de México, pp. 15-43.

Hernández Navarro, Luis (2011), No habrá recreo. (Contra-reforma constitucional y desobediencia magisterial), México, Rosa Luxemburg Stiftung/Para Leer en Libertad.

Hernstein, Richard J. y Charles Murray (1994), The bell curve. Intelligence and class structure in American life, Estados Unidos, The Free Press.

Instituto Nacional para la Evaluación de la Educación (INEE) (2012), La educación en México: estado actual y consideraciones sobre su evaluación, México, INEE.

(2015), Los docentes de México informe 2015, México, Instituto Nacional para la Evaluación de la Educación.

Illich, Iván (2015), Obras reunidas, México, Fondo de Cultura Económica.

Jiménez Gámez, Rafael (2012), «Diversidad cultural y lingüística, identidad e inmigración: algunas conclusiones y propuestas desde la investigación», Revista de Educación Inclusiva, 5(1), pp. 139-156.

Juncos, Luis Manuel (2016), Diseño e implementación de la reforma educativa como política pública. Estudio de sus efectos en la dinámica organizacional: el caso del Instituto de la Educación Básica del Estado de Morelos (tesis doctoral), México, Universidad Autónoma Metropolitana.

Martínez Rizo, Felipe (1996), La calidad de la educación en Aguascalientes. Diseño de un sistema de monitoreo, México, Universidad Autónoma de Aguascalientes/Instituto de Educación de Aguascalientes.

(2004), «Presente y futuro de la evaluación educativa en México», en Jornadas de evaluación educativa, México, Instituto Nacional para la Evaluación de la Educación, pp. 13-19. 
Mercado Maldonado, Ruth (2012), Los saberes docentes como construcción social. La enseñanza centrada en el niño, México, Fondo de Cultura Económica. Muñoz Izquierdo, Carlos (1999), Calidad de la educación. Políticas instrumentadas en diversos países para mejorarlas, México, Universidad Iberoamericana/Instituto de Fomento e Investigación Educativa/Fundación Mexicana para la Salud. Murillo Torrecilla, F. Javier, Cynthia A. Martínez Garrido y Reyes Hernández Castilla (2011), «Decálogo para una enseñanza eficaz», Revista Iberoamericana sobre Calidad, Eficacia y Cambio en Educación, 9(1), pp. 7-27.

Organización para la Cooperación y el Desarrollo Económicos (OCDE) (1991), Escuelas y calidad de la enseñanza. Informe internacional, España, Paidós. (2012), Panorama de la educación. Indicadores 2012, España, OCDE.

Padilla Arroyo, Antonio (coord.) (2012), Arquetipos, memorias y narrativas en el espejo. Infancia anormal y educación especial en los siglos XIX y XX, México, Juan Pablos Editor/Universidad Autónoma del Estado de Morelos.

Poder Ejecutivo Federal (1989), Programa para la modernización educativa 19891994, México.

Rockwell, Elsie y Rafael Quiroz (1990), «Problemas de la educación básica en México», Cero en Conducta, 5(17), pp. 25-33.

Rockwell, Elsie (2013), «La complejidad del trabajo docente y los retos de su evaluación. Resultados internacionales y procesos nacionales de reforma educativa», en La reforma constitucional en materia educativa: alcances y desafios, México, Senado de la República/Instituto Belisario Domínguez, pp. 77-110. Román, Marcela (2008), «Investigación Latinoamericana sobre enseñanza eficaz», en Eficacia escolar y factores asociados en América Latina y Caribe, Santiago de Chile, Instituto Latinoamericano de la Comunicación Educativa/Organización de las Naciones Unidas para la Educación, la Ciencia y la Cultura, pp. 209-225. 


\section{Gustavo Adolfo EnRÍQuez GutiÉRREZ}

Saint-Onge, Michel (2000), Yo explico, pero ellos... ¿aprenden?, México, Secretaría de Educación Pública/Fondo de Cultura Económica/Mensajero.

Sandoval Hernández, Andrés (9 de diciembre de 2016), «PISA 2015: todo funciona en algún lado y nada funciona en todos lados», Nexos.

Secretaría de Educación Pública (SEP) (2015a), Guía para la elaboración de la planeación didáctica argumentada. Docentes educación preescolar, México, SEP/ Coordinación Nacional del Servicio Profesional Docente.

(2015b), Perfiles, parámetros e indicadores para docentes y técnicos docentes, México, SEP/Subsecretaría de Educación Básica/Coordinación Nacional del Servicio Profesional Docente.

Schmelkes, Sylvia (1994), Hacia una mejor calidad de las escuelas, Estados Unidos, Organización de los Estados Americanos.

Sindicato Nacional de Trabajadores de la Educación/Gobierno Federal/Secretaría de Educación Pública/Secretaría de Hacienda y Crédito Público/Secretaría de Desarrollo Social/Salud (s/f), Alianza por la calidad de la educación, México, SNTE/Gobierno Federal/SEP/SHCP/Sedesol/Salud.

Skliar, Carlos (2002), ¿Y si el otro no estuviera aquí? Notas para una pedagogía (improbable) de la diferencia, Argentina, Miño y Davila.

Slee, Roger y Gaby Weiner (2001), ¿Eficacia para quién? Crítica de los movimientos de las escuelas eficaces y de la mejora escolar, España, Akal.

Stenberg, Robert J. (2003), «Cognitive contributions to learning, development, and instruction», en William M. Reynolds y Gloria E. Miller (eds.), Handbook of Psychology (volumen 7), New Jersey, John Wiley y Sons, pp. 23-122.

Tapia G., Luis Arturo y Giovanna Valentini (2015), «Desigualdad educativa y desigualdad social en México. Nuevas evidencias desde las primarias generales en los estados», Perfiles Educativos, XXXVII(151), pp. 32-54. 
Tomlinson, Carol Ann (2005), Estrategias para trabajar con la diversidad en el aula, Argentina, Paidós.

Torres, Rosa María y Emilio Tenti (2000), Políticas educativas y equidad en México. La experiencia de la educación comunitaria, la telesecundaria y los programas compensatorios, Argentina, Instituto Internacional de Planeamiento de la Educación-Organización de las Naciones Unidas para la Educación, la Ciencia y la Cultura.

Organización de las Naciones Unidas para la Educación, la Ciencia y la Cultura/ Oficina Regional de Educación (1990), «Satisfacción de las necesidades básicas de aprendizaje: una visión para el decenio del 1990» (documento de referencia), Conferencia Mundial sobre Educación para Todos, Jomtien, Chile, UNESCO/OREALC.

Organización de las Naciones Unidas para la Educación, la Ciencia y la Cultura (UNESCO) (2004), Una educación de calidad para todos los jóvenes, Suiza, UNESCO/Organización Mundial de Sanidad Animal.

Winesen, Gavin (2011), The art of gettin by (El arte de pasar de todo), Estados Unidos.

Williamson, John (1990), The Progress of Policy Reform in Latin America, Washington DC, Institute for International Economics. 